\title{
Structural and Functional Evolution of Jejunal Allograft Rejection in Rats and the Ameliorating Effects of Cyclosporine Therapy
}

\author{
James L. Madara and Robert L. Kirkman \\ Departments of Pathology and Surgery, Brigham and Women's Hospital and Harvard Medical School, and the Harvard Digestive \\ Diseases Center, Boston, Massachusetts 02115
}

\begin{abstract}
We assessed the structural and functional evolution of small intestinal transplant rejection in a rat model by use of $1-\mu \mathrm{m}$ section, electron microscopic, and in vitro electrophysiologic techniques to study jejunal mucosa 3, 6, and 9 d posttransplantation. The earliest structural abnormalities detected in jejunal loops transplanted from Lewis $\times$ Brown Norway $F 1$ hybrids into Lewis rats occurred within 3 d posttransplantation and consisted of focal endothelial cell injury of the microvasculature and focal injury of crypt epithelial cells. Both alterations were associated with adjacent infiltration of large lymphoid cells, and both markedly progressed and became rather diffuse over the following $6 \mathrm{~d}$. In contrast, villus absorptive cells were not markedly altered in structure until the 9th postoperative day. As compared with host jejuna, allograft jejunal epithelium demonstrated multiple functional abnormalities. Transepithelial resistance declined progressively by days 6 and 9 (both $P<$ 0.05), although baseline transepithelial spontaneous potential difference was only affected at day $9(P<0.01)$. Stimulated absorption by allograft jejuna, as assessed by measuring electrical response to mucosal glucose, was not significantly diminished until day $9(P<0.05)$. In contrast, stimulated secretion assessed by measurement of electrical response to serosal theophylline was diminished by day $6(P<.01)$. These data suggest that the earliest epithelial injury during rejection, as judged both structurally and functionally, occurs in the crypt and is paralleled by endothelial injury at the level of the microvasculature. Thus, the primary targets for rejection are most likely endothelial cells and crypt epithelial cells. In contrast, structural and functional impairment of villus epithelium is detectable only at substantially later times during rejection and are most likely secondary processes related to either ischemia produced by microvascular injury or decreased epithelial regenerative ability secondary to crypt injury. Last, we show that the detrimental structural and functional sequellae of jejunal transplantation across the major histocompatibility complex in this model is strikingly ameliorated with cyclosporine therapy.
\end{abstract}

\section{Introduction}

Irreversible loss of small intestinal function, although a relatively unusual occurrence, is a clinically devastating disorder. Inas-

Dr. Madara is supported, in part, by the American Gastroenterological Association/Ross Research Scholar Award. Address correspondence to Dr. Madara. 1984.

Received for publication 9 July 1984 and in revised form 22 October

J. Clin. Invest.

(c) The American Society for Clinical Investigation, Inc.

0021-9738/85/02/0502/11 $\$ 1.00$

Volume 75 , February $1985,502-512$ much as the presence of only a relatively small fraction of this organ is adequate to maintain nutrition, it seems reasonable to probe the potential feasibility of small intestinal transplantation using animal models. We have previously shown that the small intestine of Lewis $\times$ Brown Norway F1 hybrid rats displays structural features of rejection when examined $9 \mathrm{~d}$ after transplantation into Lewis rats (1). Evidence of rejection, as assessed only by light microscopic examination of paraffinembedded tissue, consisted of mononuclear cell infiltration of the intestinal wall, diminution of intestinal mucosal surface area, and epithelial cell attenuation and vacuolization (1). These structural alterations were paralleled by several functional abnormalities: spontaneous electrical potential difference, an index of baseline active transport, was diminished; electrical resistance, an index of barrier function to passive ion flow, was decreased (1). Cyclosporine largely prevented the above structural and functional sequellae of small intestine allograft rejection, suggesting that clinical consideration of small intestinal transplantation may be possible in the future. With this goal in mind, it is important to better understand the structural and functional evolution of small intestinal transplant rejection in this potentially useful animal model.

By use of $6-\mu \mathrm{m}$ paraffin-embedded sections, $1-\mu \mathrm{m}$ plasticembedded sections, and electron microscopy, we utilize this rat model to study, in detail, the structural evolution of small intestinal rejection by harvesting transplanted jejunal allografts at 3,6, and and $9 \mathrm{~d}$ after the operative procedure. We correlate alterations in mucosal structure at these time points with accompanying alterations in spontaneous transepithelial potential difference and transepithelial resistance as measured by electrophysiological techniques in vitro. We also utilize these in vitro preparations to measure electrical parameters of $\mathrm{Na}^{+}$coupled active glucose absorption (an index of villus absorptive cell function, [2]) and of active $\mathrm{Cl}^{-}$secretion (an index, at least in part, of crypt epithelial function, [2, 3]). The ameliorating effect of cyclosporine on the rejection process at $9 \mathrm{~d}$ is likewise monitored by the above structural and functional studies. Lastly, we control for potential structural and functional effects related to the surgical procedure by performing similar structural and functional studies on the host jejunum in each animal studied.

\section{Methods}

Animals. Adult male Lewis (LEW) ${ }^{1}$ and Lewis $\times$ Brown Norway F1 hybrids (LBN) that weighed between 200 and $300 \mathrm{~g}$ were obtained from Microbiological Associates, Walkersville, MD. The operative techniques used to produce LEW rats with LBN small intestinal transplants were identical to those previously described in which the

1. Abbreviations used in this paper: $I_{\mathrm{sc}}$, short circuit current; $\mathrm{LBN}$, Lewis $\times$ Brown Norway F1 hybrid rat; LEW, Lewis rat; PD, potential difference; $R$, resistance. 

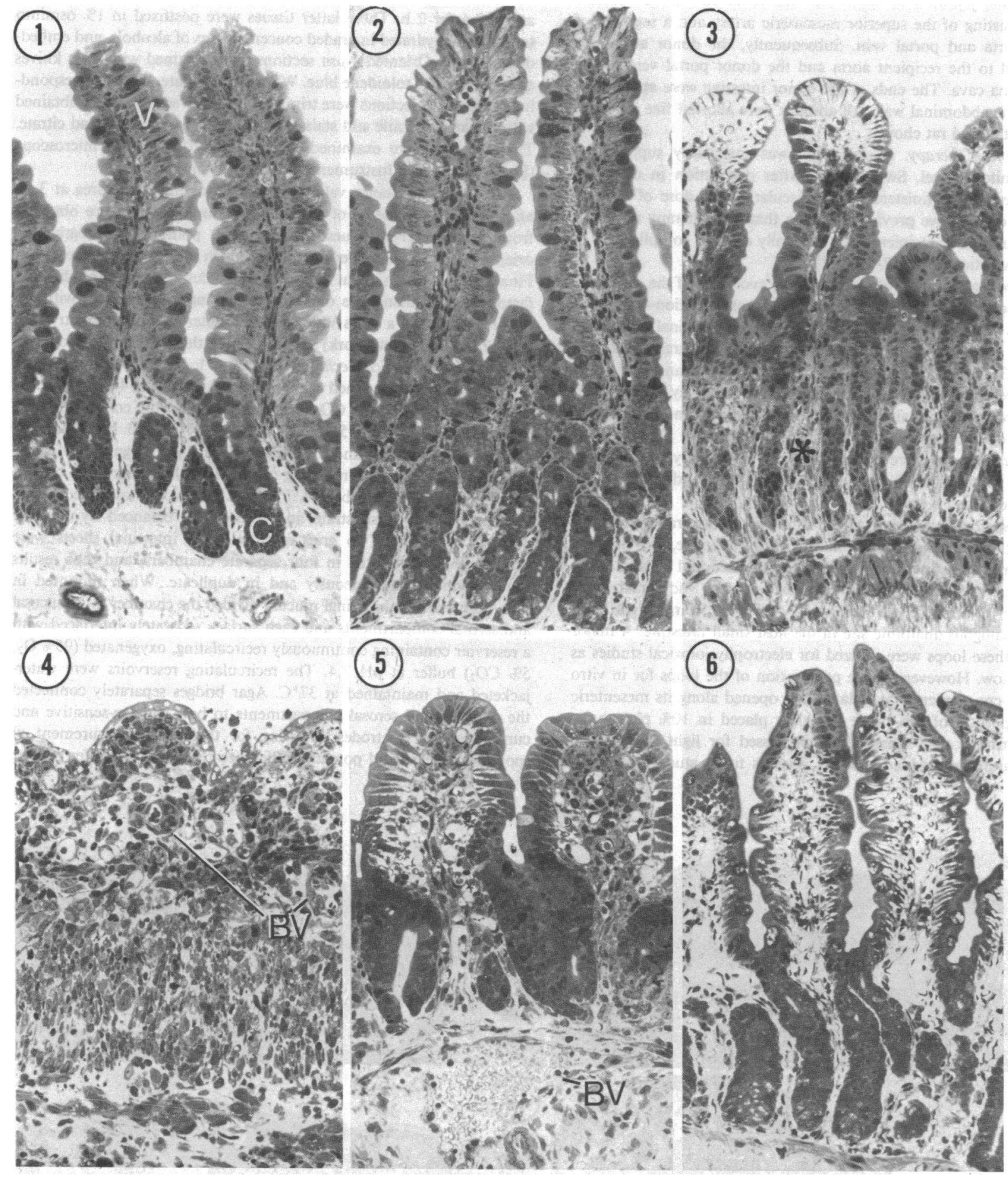

Figures 1-6. Light photomicrographs of $1-\mu \mathrm{m}$ resin-embedded sections of jejunal mucosa at various intervals after transplantation. Fig. 1: Control jejunal mucosa showing short crypts $(C)$ and tall villi $(V)$ lined by columnar villus absorptive cells. Fig. 2: Jejunal mucosa $3 \mathrm{~d}$ after transplantation. The morphologic appearance of the mucosa is similar to that of the control mucosa. Although some animal-toanimal variation in mucosal depth was noted, morphometric analysis revealed that mucosal surface amplification was not significantly different from controls. Fig. 3: Jejunal mucosa $6 \mathrm{~d}$ after transplantation. Villi are shortened and crypts appear to be minimally elongated. In addition, the paracellular spaces at the villus tips are dilated and the lamina propria adjacent to the crypt has increased numbers of mononuclear cells (*). Figs. 4 and 5: Jejunal mucosa $9 \mathrm{~d}$ after transplanta- tion. The degree of mucosal "apathy," as indicated by crypt and villus shortening, was always striking. However, the degree of cytologic abnormality was occasionally only mild (Fig. 5). Note that the more commonly seen severely affected areas with the cuboidal villus epithelial cells (Fig. 4) display fine blood vessels (BV) with compromised lumens, but the lesser affected areas (Fig. 5) have fine blood vessels which are relatively unremarkable in appearance. Fig. 6: Jejunal mucosa $9 \mathrm{~d}$ after transplantation from an animal treated with cyclosporine. The mucosal structure in cyclosporine-treated animals was remarkably preserved although morphometric analysis did reveal a substantial overall decrease in mucosal surface amplification. All $x \sim 20$; toluidene blue stain. 
host intestine remains intact (1). Briefly, the donor small intestine from proximal jejunum to midileum was removed with a vascular pedicle consisting of the superior mesenteric artery and a segment of both the aorta and portal vein. Subsequently, the donor aorta was anastamosed to the recipient aorta and the donor portal vein to the recipient vena cava. The ends of the donor intestine were attached as stomas to the abdominal wall. All animals were allowed free access to water and standard rat chow.

Cyclosporine therapy. Cyclosporine was generously supplied by Sandoz Limited (Basel, Switzerland). After dissolution in olive oil, cyclosporine was administered intramuscularly at a dose of $15 \mathrm{mg} /$ $\mathrm{kg} \cdot \mathrm{d}$ for $7 \mathrm{~d}$. We have previously shown that such therapy results in indefinite survival of recipients that normally die of a complication of transplant rejection within 7-10 d (1).

Experimental designs. (a) Studies of the evolution of the structural and functional sequellae of small intestinal transplantation-for these experiments four to five LEW rats with LBN small intestinal transplants were killed at each of the following times after the transplantation procedure: 3, 6, and $9 \mathrm{~d}$. Additionally, four LBN rats and four LEW rats that were not surgically manipulated in any way were killed. Tissues from these animals were handled as outlined in detail below. (b) Effects of cyclosporine therapy on rejection-five animals with intestinal transplants were given cyclosporine therapy, as outlined above, and were killed $9 \mathrm{~d}$ after transplantation and tissues were handled as outlined below.

Morphological techniques. At the time of sacrifice, rats were anesthetized with ether, a midabdominal incision was made, and the host and graft small intestine were completely visualized and isolated while still attached to their respective vascular pedicles. Midjejunal loops measuring $\sim 10 \mathrm{~cm}$ in length were rapidly removed from the graft and from the analogous anatomic site in the host small intestine. A major portion of these loops were utilized for electrophysiological studies as outlined below. However, before preparation of the loops for in vitro studies, a 2-cm segment was isolated and opened along its mesenteric border. A portion of this tissue was then placed in $10 \%$ phosphatebuffered formalin and subsequently processed for light microscopic study of paraffin-embedded sections. Tissues to be studied by $1-\mu \mathrm{m}$ section and electron microscopy were submerged in a $4^{\circ} \mathrm{C}$ solution of $2 \%$ formaldehyde, $2.5 \%$ glutaraldehyde in $0.1 \mathrm{M} \mathrm{Na}$ cacodylate buffer at $\mathrm{pH} 7.4$ for $2 \mathrm{~h}$. These latter tissues were postfixed in $1 \%$ osmium tetroxide, dehydrated in graded concentrations of alcohols, and embedded in Epon. Oriented $1-\mu \mathrm{m}$ sections were obtained with glass knives and stained with toluidene blue. Where appropriate, blocks corresponding to the $1-\mu \mathrm{m}$ sections were trimmed, and thin sections were obtained with a diamond knife and stained with uranyl acetate and lead citrate. Thin sections were examined in a Philips 201 electron microscope (Philips Electronic Instruments, Inc., Mahwah, NJ).

To correct resistance values for alterations in surface area at 3,6, and $9 \mathrm{~d}$, measurements of surface area amplification were obtained from representative tissues at each time point by photographing oriented paraffin-embedded sections through a light photomicroscope. Final prints were made at $\times 300$. The mucosal/serosal amplification factor for each tissue was obtained by tracing both surfaces with the magnetic stylus of a Zeiss Videoplan computerized morphometry unit (Carl Zeiss, Inc., New York) and expressing the results as a fraction.

Electrophysiological techniques. Isolated loops from both host and graft intestine were washed with a buffer solution at $4^{\circ} \mathrm{C}$ which was gassed with $95 \% \mathrm{O}_{2}, 5 \% \mathrm{CO}_{2}$, and had the following composition (in millimolar): $114 \mathrm{NaCl}, 5 \mathrm{KCl}, 1.65 \mathrm{Na}_{2} \mathrm{H} \mathrm{PO}, 0.3 \mathrm{NaH}_{2} \mathrm{PO}_{4}, 25$ $\mathrm{NaHCO}_{3}, 1.25 \mathrm{CaCl}_{2}$, and $1.1 \mathrm{MgSO}_{4}$. Unstripped segments of small intestine were mounted in Ussing chambers with surface areas of 0.64 $\mathrm{cm}^{2}$ as previously described $(4,5) .20 \mathrm{mM}$ glucose was added to the serosal reservoir as substrate and osmotically balanced by $20 \mathrm{mM}$ mucosal mannitol. Two graft and two host intestinal sheets were simultaneously mounted in four separate chambers, and thus results were obtained simultaneously and in duplicate. When mounted in such chambers, the intestinal mucosa divided the chamber into mucosal and serosal compartments and each surface separately interfaced with a reservoir containing continuously recirculating, oxygenated $\left(95 \% \mathrm{O}_{2}\right.$, $5 \% \mathrm{CO}_{2}$ ) buffer at $\mathrm{pH}$ 7.4. The recirculating reservoirs were waterjacketed and maintained at $37^{\circ} \mathrm{C}$. Agar bridges separately connected the mucosal and serosal compartments to both voltage-sensitive and current-passing electrodes, allowing for the direct measurement of spontaneous electrical potential difference (PD), and PD after passing
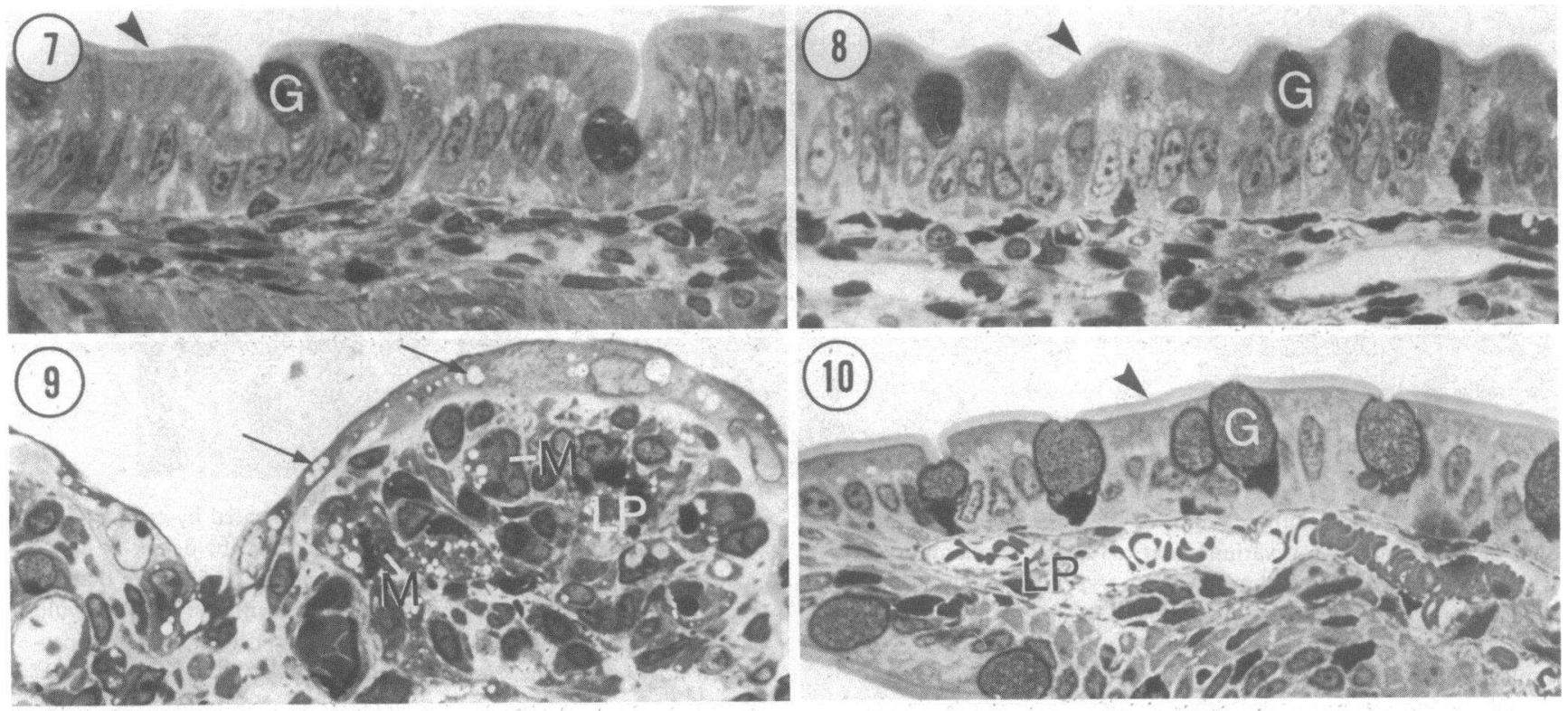

Figures 7-10. Light photomicrographs of $1-\mu \mathrm{m}$ sections of jejunal villus epithelium from control (Fig. 7), 3-d grafted (Fig. 8), 9-d grafted (Fig. 9), and 9-d grafted cyclosporine-treated (Fig. 10) jejunal loops. Tall villus epithelial cells with prominent microvillus brush border (small arrowheads) and interspersed goblet cells $(G)$ are seen in control, 3-d, and cyclosporine-treated animals. In contrast, 9-d

graft mucosa from animals untreated with cyclosporine predominantly displayed cuboidal surface epithelium, which was finely vacuolated (arrows). The lamina propria ( $L P$ ) contained scattered small lymphocytes and plasma cells in control, 3-d, and 9-d treated animals but contained many large mononuclear cells $(M)$ in 9-d untreated animals. All $\times \sim 400$; toluidene blue stain. 
a known quantity of current, and thus for the measurement of resistance $(R)$ and short circuit current $\left(I_{s c}\right)$ as calculated using Ohm's law. In each chamber before each experiment, fluid resistance was measured and subsequently subtracted from resistance values to obtain true tissue resistance.

Measurements of spontaneous PD, $R$, and $I_{\mathrm{sc}}$ were obtained after a 15 -min equilibration period. $\mathrm{Na}^{+}$-dependent active glucose absorption was assessed by measuring the peak PD and $I_{\mathbf{s c}}$ responses after exposure of the mucosal surface to $20 \mathrm{mM}$ glucose. Active $\mathrm{Cl}^{-}$secretion was assessed by measuring the peak PD and $I_{\mathrm{sc}}$ responses after the addition of $5 \mathrm{mM}$ of the phosphodiesterase inhibitor, theophylline, to the serosal reservoir. The above electrical responses to these agents are widely used as indices of these specific active transport processes $(2,6$, 7). Data were obtained from a digital recorder and statistical analyses were performed by using Student's two-tailed $t$ test. All data are expressed as means \pm standard errors.

Epithelial function and species differences. We have previously described the morphologic and functional similarity between normal LBN and LEW jejuna and the similarity between normal LEW jejuna and LEW jejunal isografts $9 \mathrm{~d}$ after transplantation (1). Thus alterations seen in LBN jejunal transplants in LEW rats as compared with LEW host jejuna within this 9-d period do not result from species differences in baseline jejunal structure and function.

\section{Results}

Morphology of the epithelium-evolution of rejection. The jejunal mucosal structure of normal LBN and LEW rats is similar to that of other mammalian species $(1,8)$ and consists of tall slender villi and short crypts with a villus/crypt height ratio of $\sim 4: 1$ (Fig. 1). Villi are lined by columnar absorptive cells with a prominent microvillus brush border and have interspersed goblet cells and occasional intraepithelial lymphoid cells (Fig. 7). Crypts are lined predominantly by low columnar to cuboidal undifferentiated crypt cells that display occasional mitotic figures and have a microvillus border, which is relatively inapparent by light microscopy (see Fig. 13). Other specialized cells, such as goblet cells, Paneth cells, and endocrine cells, are also scattered throughout the crypt epithelium. The general structural features of the mucosa were maintained through the 3rd d after transplantation (Fig. 2), and there was no evidence of villus epithelial cell damage as judged by light microscopy (Fig. 8). The ultrastructural appearance of villus epithelium 3 d posttransplant was also comparable with that of normal villus epithelium. In contrast to the normal light microscopic appearance of villus epithelium in 3-d allografts, rare foci of crypt epithelial cell damage were found in $1-\mu \mathrm{m}$ sections of these tissues (Fig. 14). These consisted of isolated crypts lined by cells with lucent finely vacuolated cytoplasm and enlarged lucent nuclei (Fig. 14). Electron microscopic analysis of such areas yielded little additional structural information, merely showing damaged undifferentiated crypt cells with vacuolated, electron lucent cytoplasm. The surrounding lamina propria at such sites was enriched in large lymphoid cells (Fig. 14). An alteration in mucosal/serosal surface amplification was not subjectively obvious at day 3, and morphometric analysis revealed little change in mean amplification (10.9 vs. 10.2, for control vs. 3-d allografts, respectively).

By day 6 shortening of villi could be readily appreciated by light microscopy (Fig. 3). Quantitative evaluation revealed a substantial decrease in mucosal/serosal surface amplification (10.9 vs. 6.5 for control vs. $6-\mathrm{d}$ allografts, respectively). However, as judged in $1-\mu \mathrm{m}$ sections and thin sections, villi were still lined by columnar absorptive cells with prominent brush borders that were not damaged and, by all structural parameters,
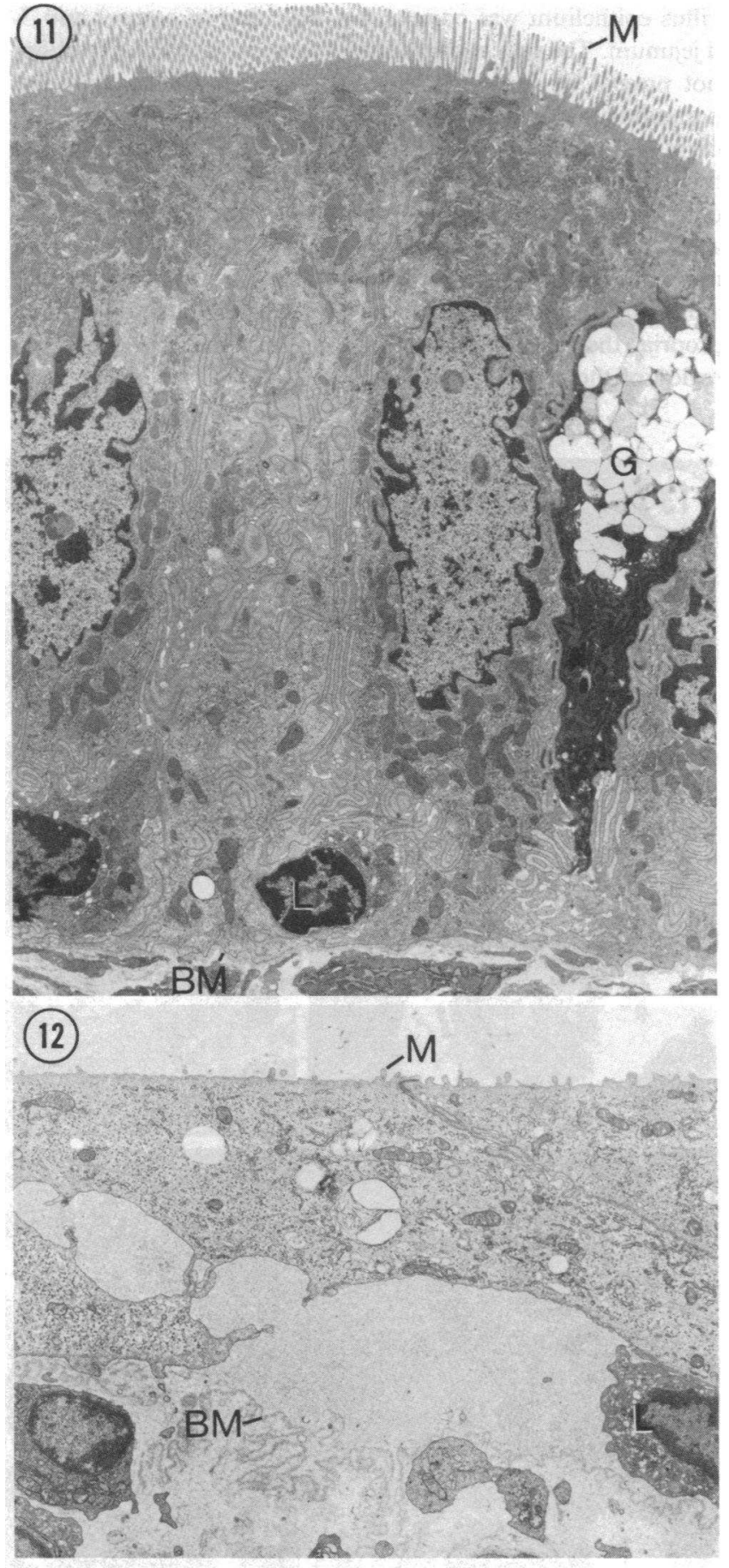

Figures 11 and 12. Electron photomicrographs depicting the remarkable preservation of villus epithelial cell structure with cyclosporine. Fig. 11: Representative villus absorptive cells with an interspersed goblet cell $(G C)$ from graft jejunum 9-d after transplantation with cyclosporine therapy. Absorptive cells have remarkably preserved structure and display a tall, lush, regular microvillus brush border $(M)$. The cells maintain their association with the basement membrane $(B M)$. A small intraepithelial lymphocyte is present $(L)$. Fig. 12: Representative villus absorptive cells from graft jejunum $9 \mathrm{~d}$ after transplantation without cyclosporine therapy. The absorptive cells are cuboidal and vacuolated and show extremely sparse, shortened, and irregular microvilli $(M)$. Focally the epithelial cells are detached from the basement membrane $(B M)$. As in all intestinal epithelium, occasional small intraepithelial lymphocytes $(L)$ are present. Differentiated goblet cells were not seen. Both $\times \sim 7,000$; uranyl acetate, lead citrate stain. 
villus epithelium was comparable with that of control and 3d jejunum. The only morphologic alteration of villus epithelium not present in controls was focal dilatation of the epithelial paracellular spaces at the tips of villi (Fig. 3). In contrast, crypts appeared to be mildly elongated (Fig. 3) and, in scattered foci, displayed evidence of epithelial damage such as that described, but more infrequently present, at $3 \mathrm{~d}$ (see Figs. 15 and 16). The lamina propria at day 6 was distended by an infiltrate consisting predominantly of large lymphoid cells and, although these cells were scattered throughout the lamina propria, the density of this infiltrate appeared greater in the region of the crypt (Fig. 3).
At $9 \mathrm{~d}$ the serosal/mucosal surface amplification was severely diminished (10.9 vs. 2.6 for control and 9-d allografts, respectively), and the epithelium was extensively damaged (Figs. 4 and 5). Characteristically, villus epithelial cells were markedly foreshortened and did not display a prominent brush border (Fig. 4). However, extensive sampling of $1-\mu \mathrm{m}$ sections revealed some variation in the degree of damage-although jejunal mucosal structure was always simplified, in occasional foci attenuated villi were lined by columnar absorptive cells with prominent brush borders (Fig. 5). Even in areas of extreme damage, an unequivocal increase in villus intraepithelial lymphoid cells was not detectable. In contrast, the lamina propria
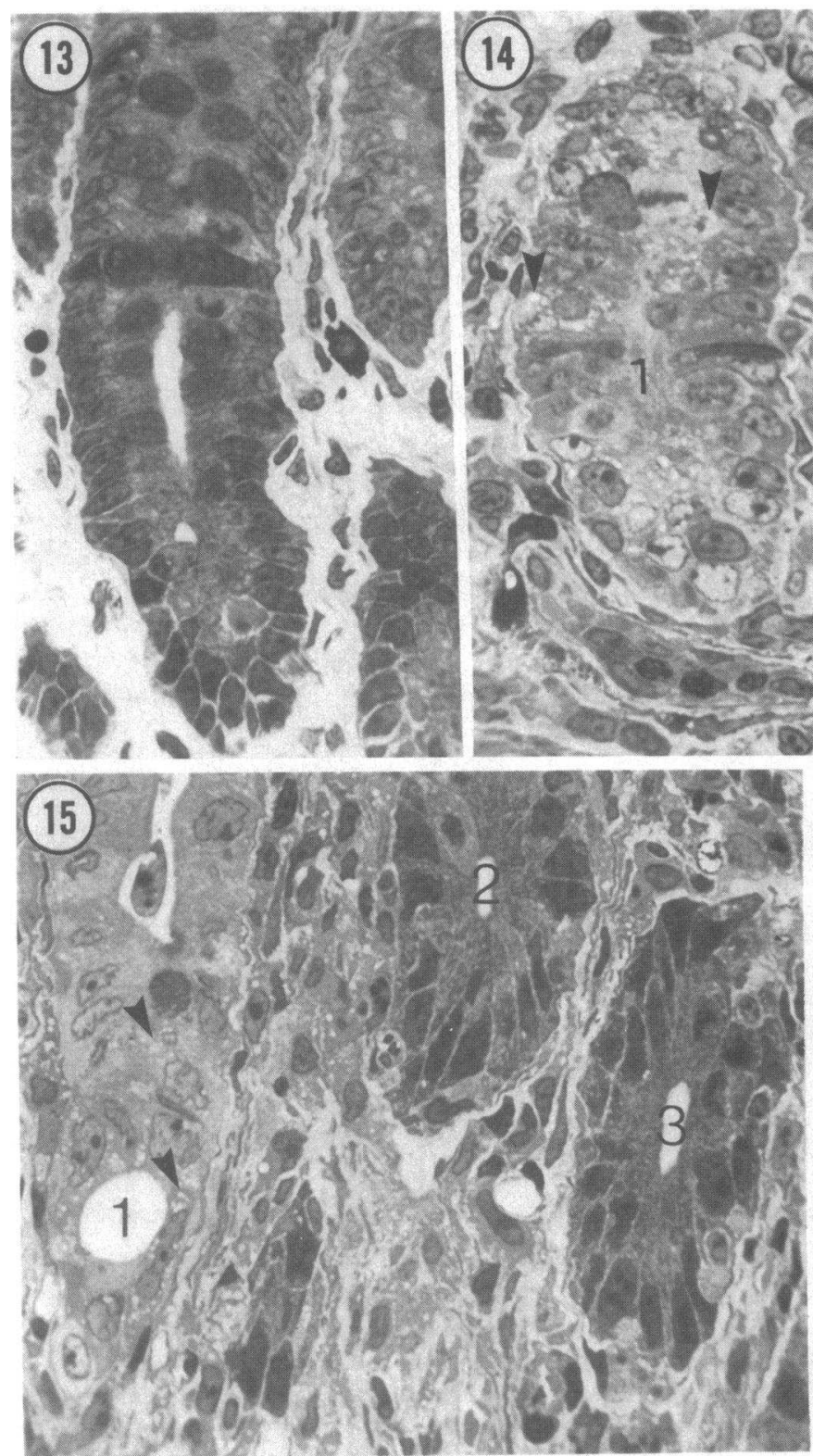

Figures 13-16. Light photomicrographs of 1- $\mu \mathrm{m}$ sections of crypt epithelium from control (Fig. 13), 3-d grafted (Fig. 14) and 6-d grafted (Figs. 15 and 16) jejunal loops. Fig. 13: In controls, crypts are lined by cuboidal epithelial cells of constant height and cytoplasmic density. Fig. 14: By $3 \mathrm{~d}$ posttransplant grafted loops showed rare foci (crypt l), in which crypt epithelium was extensively vacuolated (arrowheads) and displayed lucent cytoplasm. Such foci were surrounded by a lamina propria infiltrate of large mononuclear cells, and adjacent crypts (crypt 2) often displayed no such evidence of
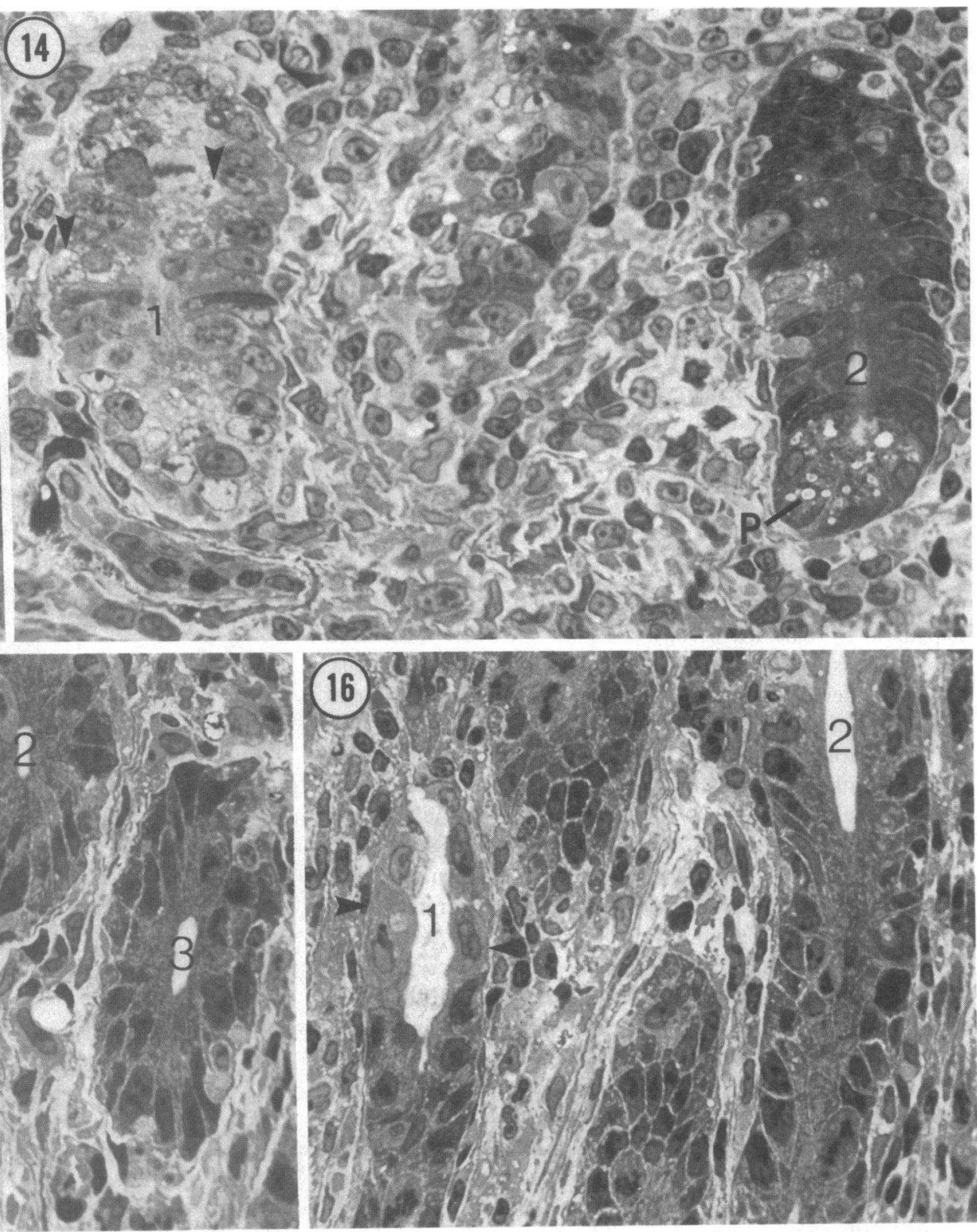

cellular injury. The vacuoles present at the base of crypt 2 are Paneth cell granules and do not represent an abnormality. Figs. 15 and 16: In grafts at $6 \mathrm{~d}$, damaged crypts were still focally present but much easier to find than in 3-d grafts. Damage consisted of foci displaying lucent vacuolated cells (crypts labeled 1) and occasionally shortening of undifferentiated crypt cells (Fig. 16, crypt 1). Such foci were interspersed with crypts containing epithelial cells with normal structure (crypts labeled 2 and 3). All $\times \sim 40$; toluidene blue stain. 


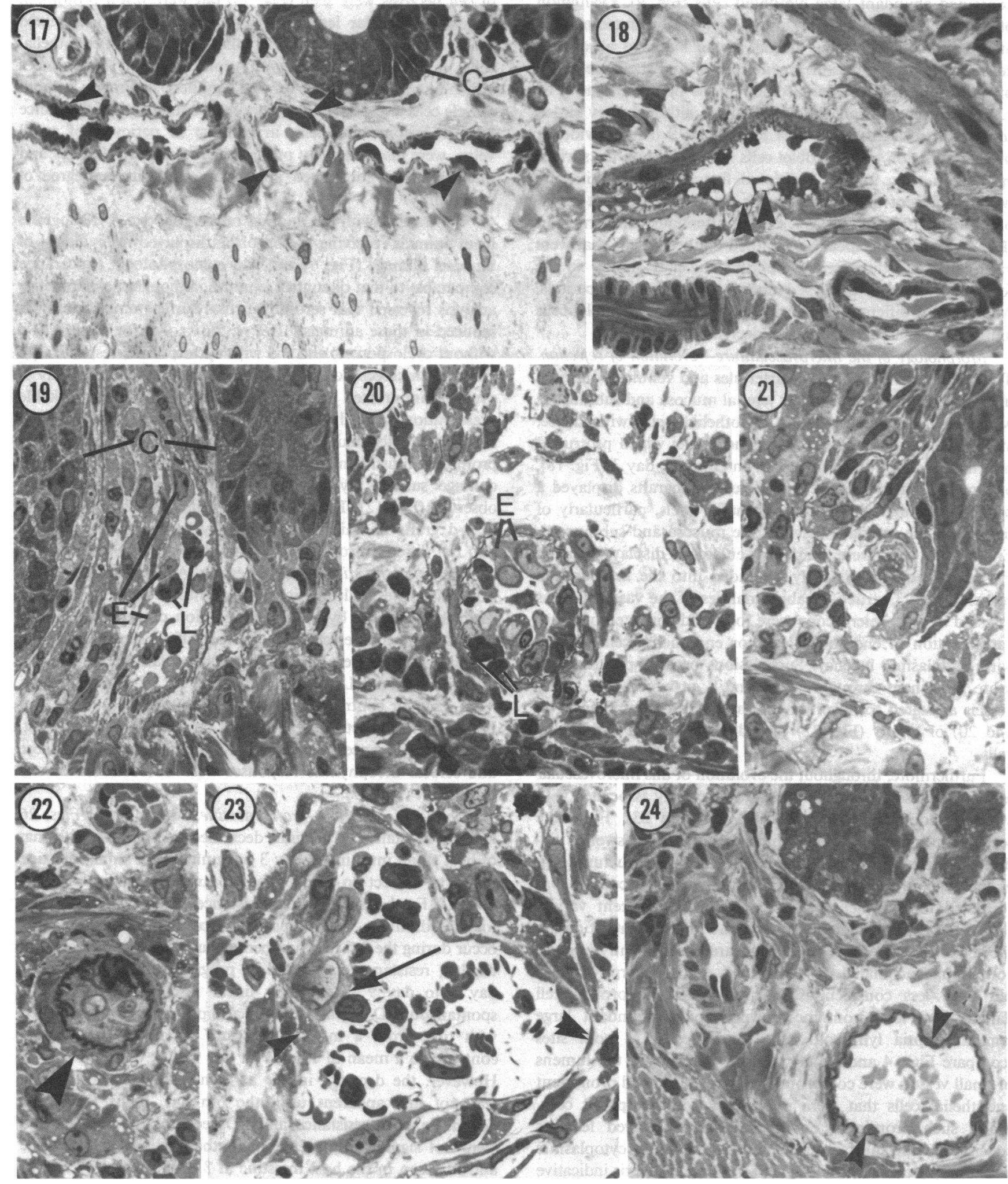

Figures 17-24. Light photomicrographs of $1-\mu \mathrm{m}$ sections showing alterations of the microvasculature occurring in transplanted jejuna. Fig. 17: Control jejunum displayed microvasculature lined by endothelial cells, which had prominent nuclei (arrowheads) jutting into the lumen but attenuated cytoplasmic processes that were difficult to detect by light microscopy. The base of crypts $(C)$ are present in the upper portion of the plate. Fig. 18: By $3 \mathrm{~d}$ grafts revealed foci where small vessels had either swollen endothelial cells or vacuolated endo- thelial cells (arrowheads). Fig. 19: 6-d graft displaying a venule with prominent swollen endothelial cells $(E)$ with closely associated lymphoid cells $(L)$. Crypts $(C)$ are present on either side of this vessel. Fig. 20: 9-d graft with arteriole showing a lumen, which is markedly attenuated by swollen endothelial cells displaying lucent cytoplasm and having intermixed large lymphoid cells $(L)$. Fig. 21: At $9 \mathrm{~d}$ small vessels multifocally demonstrated necrotic foci with fibrinlike depositions (arrowheads). Fig. 22: 9-d graft arteriole (arrowhead) with a 
contained abundant large lymphoid cells (Fig. 9). Evaluation of absorptive cells by electron microscopy demonstrated marked attenuation of microvillus height in the flattened absorptive cells as well as cytoplasmic vacuolization and the appearance of myelinlike intracellular accumulations of membrane (Fig. 12). Such changes were diffusely present, although they varied somewhat in degree from area to area. At $9 \mathrm{~d}$ little evidence of differentiation toward goblet cells was present on the surface epithelium (Fig. 9). An additional feature seen multifocally was detachment of absorptive cells from the basement membrane (Fig. 12). Crypts at $9 \mathrm{~d}$ were attenuated and irregular (Figs. 4 and 9) and focally contained epithelial cells that were vacuolated and displayed cytoplasmic lucency similar to the focal crypt lesions described above at earlier time periods. Additionally, differentiated crypt cells such as crypt goblet cells and Paneth cells were markedly decreased in number.

Morphology of the microvasculature-evolution of rejection. The fine vessels including the arterioles and venules of normal unoperated control and of host jejunal mucosa and submucosa were lined by inconspicuous endothelial cells with dense, attenuated cytoplasmic processes and nuclei which protruded into the vascular lumen (Fig. 17). In contrast, day 3 (Fig. 18), 6 (Fig. 19), and 9 (Figs. 20-23) jejunal allografts displayed a prominent progressive lesion of small vessels, particularly of those near the interface between the mucosa and submucosa. Endothelial cells were markedly enlarged, displayed lucent cytoplasm, and prominently distended into the intraluminal space. At day 3, structural abnormalities of the vasculature as assessed by light microscopy were focal and consisted of either vacuolization of endothelial cells or endothelial cell swelling with cytoplasmic lucency. Such lesions became progressively more prominent through days 6 and 9, at which time many vessels appeared to have lumens that were partially (Figs. 19 and 20) or totally (Fig. 22) occluded by swollen endothelial cells.

Furthermore, throughout the evolution of this microvascular lesion intravascular lymphoid cells were closely associated with swollen endothelial cells (Figs. 19, 20, and 23). Fortunate sections of affected vessels, which included both swollen endothelial cells and endothelial cells of normal structure, suggested that direct lymphoid cell-endothelial cell contact was restricted to endothelial cells of the swollen, lucent variety (Fig. 23). Of particular interest was the finding that, at day 9, the above lesions of fine vessels appeared prominently in areas containing vacuolated, foreshortened villus absorptive cells, but were less conspicuous in areas where absorptive cell damage was less pronounced even though abundant large lamina propria lymphoid cells were present at both sites (compare Figs. 4 and 5). By electron microscopy the lumens of small vessels were compromised by the distended prominent endothelial cells that were intermixed with lymphoid cells. Many such prominent endothelial cells displayed features consistent with substantial cell injury including cytoplasmic lucency and vacuolization (Fig. 25). Foci of cell debris indicative of cell necrosis were seen (Fig. 25) but were not a prominent feature of vascular lesions. Lymphoid cells were intermixed within the vessel walls and adherent to the surface of endothelial cells. Pericytes were also very prominent and displayed a hobnail type appearance around venules. Arteriolar smooth muscle cells also displayed a prominent hobnail type appearance in such lesions but these, in contrast to pericytes of small veins, were also relatively prominent in control arterioles and thus could be considered abnormal only with a degree of uncertainty.

Contrasting with the findings outlined above, 9-d allografts from animals receiving cyclosporine displayed no evidence of mucosal damage (Fig. 6) and had a morphologic appearance comparable to that of control jejunum, although morphometric analysis revealed that serosal/mucosal surface amplification is reduced in these animals (10.9 vs. 2.6 vs. 8.0 for control, $9 \mathrm{~d}$ without cyclosporine, and $9 \mathrm{~d}$ with cyclosporine, respectively). Electron microscopic examination of the epithelium of day 9 , cyclosporine-treated transplanted jejuna showed remarkable preservation of cellular architecture (Fig. 12). Specifically, absorptive cells maintained a columnar appearance with a prominent brush border, and no structural evidence of epithelial damage such as cytoplasmic vacuolization or lucency was observed (Fig. 12). The structure of small vessels of the jejuna in 9-d grafts of animals receiving cyclosporine therapy was also not different from that in normal controls (Fig. 24).

Epithelial function-host intestine. As shown in Fig. 26, host small intestinal function appeared to be deleteriously, but reversibly, affected by the transplantation procedure. Notably both spontaneous electrical potential difference and transepithelial resistance were decreased in host small intestine at $3 \mathrm{~d}$ as compared with LEW controls ( $\bar{x}: 2.6$ vs. $1.7 \mathrm{mV}$ and 61 vs. $41 \Omega \cdot \mathrm{cm}^{2}$ for control and 3-d host jejunum respectively), but both parameters returned to control range by $9 \mathrm{~d}$. In contrast, significant differences between control and host mucosal glucose and serosal theophylline responses were not detected at any time point (Fig. 27).

Epithelial function-graft intestine. As shown in Figs. 26 and 28 , resistance of the graft decreased comparably with that of host small intestine at day 3 but unlike host small intestine failed to correct to unoperated control levels by day 9 ( $P$ $<0.01)$. Furthermore, when resistance values were corrected for the dramatic alterations in mucosal amplification that occur during the 9-d rejection period studied (see morphological results), resistance was found to progressively decrease from day 3 to day 9 in a nearly linear fashion (Fig. 28). The spontaneous PD of graft intestine also progressively diminished with time from a mean of $2.8 \mathrm{mV}$ at day 0 (unoperated controls) to a mean of $1.8 \mathrm{mV}$ at day $9(P<0.001)$ (Fig. 29). However, the decrease in PD as compared with host jejuna was not first apparent until the 6th day. Thus the initial decline in both resistance and PD seen in the grafts at $3 \mathrm{~d}$ were not significantly greater than the declines in resistance and PD seen in the host intestine at $3 \mathrm{~d}$. However, by $9 \mathrm{~d}$, as lumen, which has been occluded due to marked endothelial cell swelling. Fig. 23: In fortunate planes of section of vessels from 6- and 9-d grafts that displayed areas containing both prominent swollen endothelial cells and shortened attenuated endothelial cells that were similar to those in controls (double arrowhead); the swollen endothelial cells were more frequently intimately associated with lymphoid cells (arrow) Fig. 24: The microvasculature from 9-d grafts treated with cyclosporine was free of the vascular lesions depicted above. Rather, endothelial cells with prominent nuclei protruding into vascular lumen (arrowheads) but with dense, attenuated cytoplasmic processes were present, and thus such vessels were similar to those of control jejuna. All $\times \sim 400$; toluidene blue stain. 


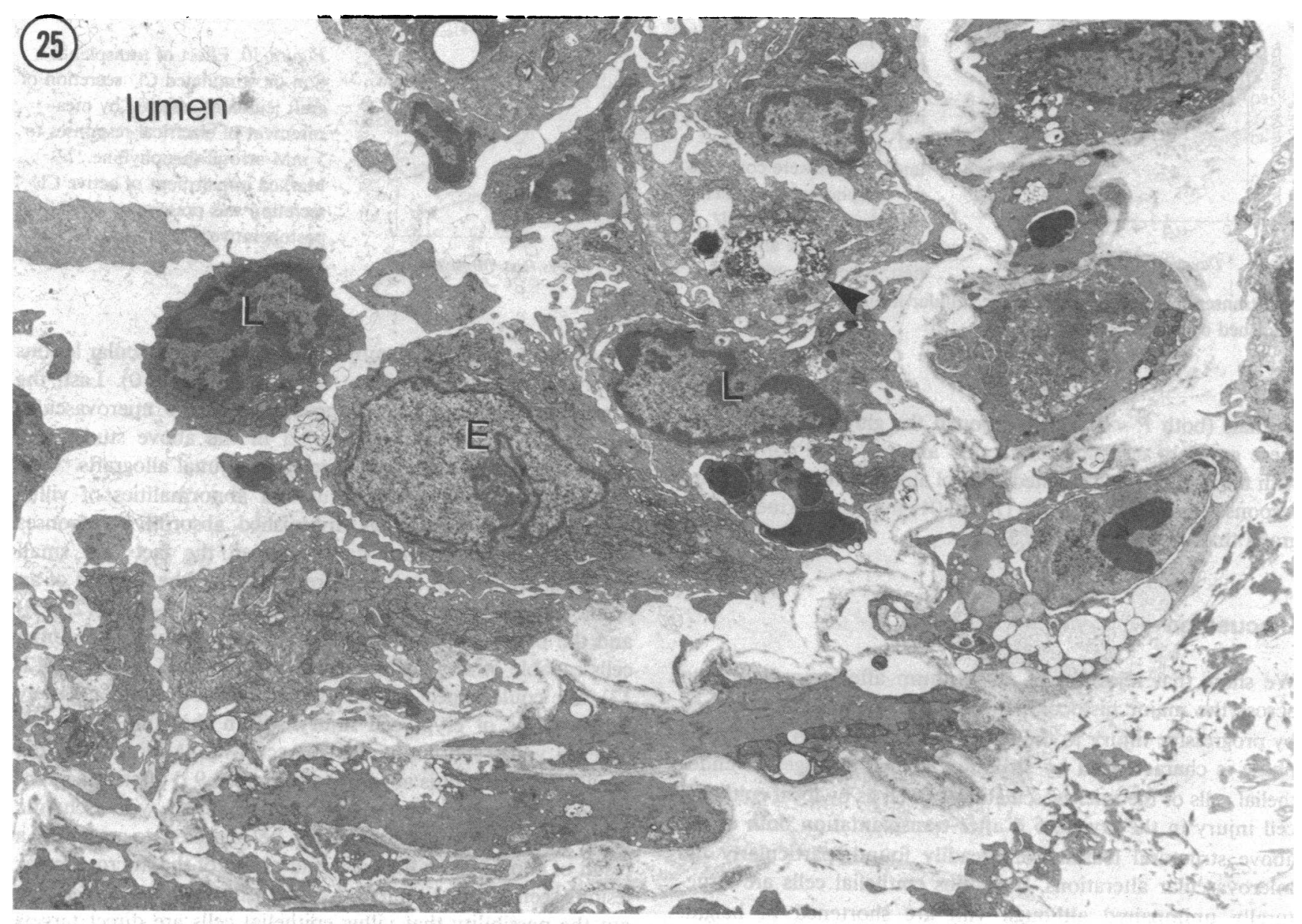

Figure 25. Electron photomicrograph showing the wall of a small mucosal vessel in a 9-d graft from an animal untreated with cyclosporine. Endothelial cells $(E)$ display electron-lucent cytoplasm, are bulging into the lumen, and are markedly compromising lumenal

the host values returned toward control values, clear differences were apparent between host and graft bowel for both spontaneous $\mathrm{PD}(P<0.001)$ and $R(P<0.01) . I_{\mathrm{sc}}$ in grafts appeared to rise (NS) initially at day 3 and subsequently and progressively diminished until, at day $9, I_{\mathrm{sc}}$ was less than control values $(P$ $<0.05$ ) (Fig. 29).

Parameters of stimulated active secretion were markedly impaired by the rejection process. As shown in Fig. 30, both $P D$ and $I_{s c}$ response to $5 \mathrm{mM}$ serosal theophylline were less (both $P<0.01$ ) than host values (Fig. 27) by day 6 . In contrast,

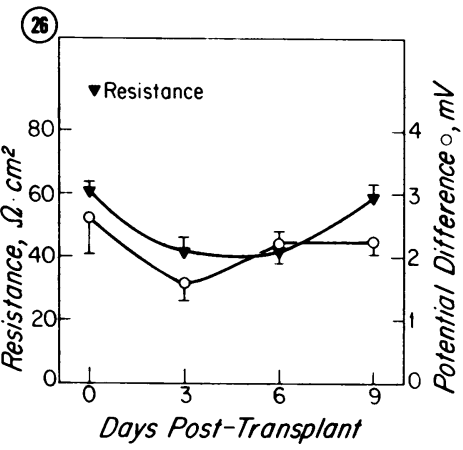

Figure 26. Effects of transplantation on baseline functional parameters of host jejunum as assessed in vitro. Both resistance and potential difference decreased by $3 \mathrm{~d}$ in host jejunum as compared with control jejunum but by $6 \mathrm{~d}$ (PD) or $9 \mathrm{~d}$ (Resistance) had returned to control values. surface area. Multiple lymphocytes $(L)$ are present in the wall of the vessel. A focus of cellular debris and multilamellar membrane suggestive of cell necrosis is present within the wall (arrowhead). $\times \sim 7,500$; uranyl acetate, lead citrate stain.

the PD and $I_{\mathrm{sc}}$ responses of graft epithelium to $20 \mathrm{mM}$ mucosal glucose were not significantly different from host responses until $9 \mathrm{~d}$ (Figs. 27 and 31) (both $P<0.05$ ).

Epithelial function-modulation with cyclosporine. We have previously shown that cyclosporine partially prevents the fall in PD and resistance (corrected for surface area) which accompanies rejection (1). As shown in Fig. 32, stimulated $\mathrm{Cl}^{-}$ secretion (theophylline response) and stimulated $\mathrm{Na}^{+}$-glucose coupled absorption are also greater in 9-d grafts of animals treated with cyclosporine than in 9-d grafts from untreated

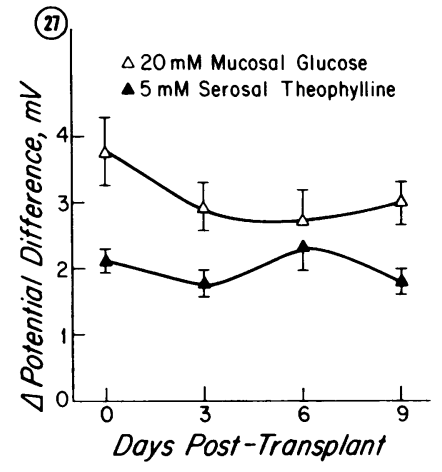

Figure 27. Effects of transplantation on parameters of stimulated active transport in host small intestine. $\mathrm{Na}^{+}$-coupled active glucose absorption as measured by PD response to $20 \mathrm{mM}$ mucosal glucose and active $\mathrm{Cl}^{-}$secretion as measured by PD response to 5 $\mathrm{mM}$ serosal theophylline were not significantly altered by transplantation surgery at any time point studied. 


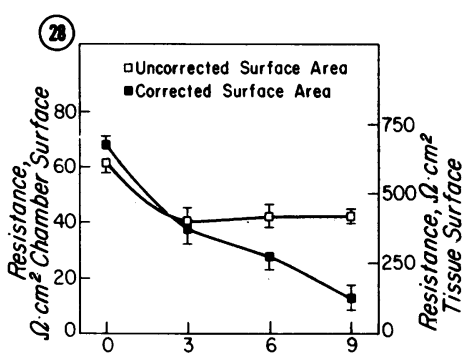

Days Post-Transplant
Figure 28. The effect of transplantation on graft transepithelial resistance. Measured resistance of graft intestine fell by $3 \mathrm{~d}$ but remained unaffected through $9 \mathrm{~d}$. However, when resistance values were corrected for morphometrically determined values of serosal/ mucosal surface amplification, it became apparent

that transepithelial resistance, at the cellular level, progressively diminished over the 9-d period.

animals (both $P<0.005$ ). Although the glucose responses in these grafts of cyclosporine-treated animals were comparable with those of nontransplanted control animals, the $\mathrm{Cl}^{-}$secretory response was actually greater $(P<0.01)$ in cyclosporine-treated grafts than in controls (Fig. 32).

\section{Discussion}

We show that rejection of rat jejunum after transplantation across the major histocompatibility complex is characterized by progressive injury to the jejunal mucosa, which in its early phase is characterized by striking cytologic changes of endothelial cells of the microvasculature as well as by focal epithelial cell injury in the crypts. $6 \mathrm{~d}$ after transplantation both of the above structural features are readily found, particularly the microvascular alterations, but villus epithelial cells are structurally unimpaired although villi are shortened in height. Although it is uncertain whether the initial site of rejection in jejunal grafts is epithelial, endothelial, or both, the above sequential observations suggest that the endothelium and perhaps the crypt epithelium are the initial sites of injury. It is important to stress however that although we find no structural evidence indicative of a primary response against villus epithelium, this finding does not definitively rule out the possibility that villus epithelium could also be a primary target for rejection.

The concept that endothelial cells represent a major targeted site for transplant rejection has also been suggested based on structural studies of experimental heart (9), skin (10), and renal (11) allografts. Indeed, the vascular endothelium of cardiac allografts loses its ability to restrict transendothelial macromolecular flow before structural injury to myocytes occurs (9). Similarly, studies of first set rejection of skin

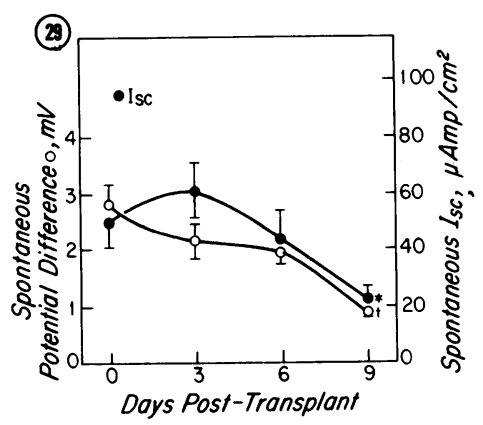

Figure 29. Effect of transplantation on graft baseline PD and $I_{s c} . I_{s c}$ and PD did not significantly change until $9 \mathrm{~d}$ after transplantation.

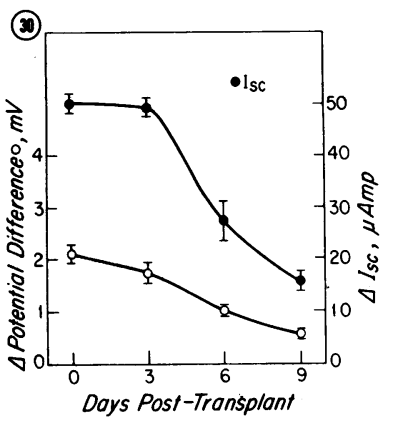

Figure 30. Effect of transplantation on stimulated $\mathrm{Cl}^{-}$secretion of graft jejuna as assessed by measurement of electrical responses to $5 \mathrm{mM}$ serosal theophylline. Marked impairment of active $\mathrm{Cl}^{-}$ secretion was present by the 6 th postoperative day in graft jejunum.

allografts in man have demonstrated that microvascular lesions invariably predate significant epithelial injury (10). Last, the time course and structural appearance of the microvascular endothelial abnormalities reported in the above studies are comparable to our findings in rejected jejunal allografts.

Consistently detectable structural abnormalities of villus absorptive cells as well as diminished absorptive responses were not apparent until day 9. Given the fact that small intestinal epithelium is rapidly regenerating (the lifespan of an epithelial cell, once on the villus, is only $\sim 3-5 \mathrm{~d}[12,13])$ and the only compartment within this epithelium containing cells capable of moving through the cell cycle is the crypt (12, 13), one could expect a diminished villus compartment solely on the basis of crypt cell injury within the time frame we observe villus shortening to be occurring. Additionally, the severe injury to villus absorptive cells seen $9 \mathrm{~d}$ after transplantation is nonspecific in nature but consistent with ischemic injury (14) and might reasonably be expected to occur as a result of anoxia given the profound extent of the microvascular lesions seen at this time point. Thus, although we cannot rule out the possibility that villus epithelial cells are direct targets of the rejection process, based on the absence of such injury at early time points and the ease with which such injury at later time points can be explained as secondary to other processes, we speculate that villus epithelium is not a direct target of the rejection process. In contrast, our findings suggest that crypt epithelium may be a primary target of the rejection process given the early time point at which crypt injury can be found. The suggestion that similar scattered crypt injury is the earliest structural lesion found in the intestine during graft vs. host disease $(15,16)$ is of interest in this regard. We stress that the proposed series of events outlined above is speculative and based on our serial, detailed observations of mucosal structure.

We additionally demonstrate a deterioration in the ability of jejunal allografts to resist passive transepithelial flow of ions as assessed by measurements of electrical resistance in vitro.

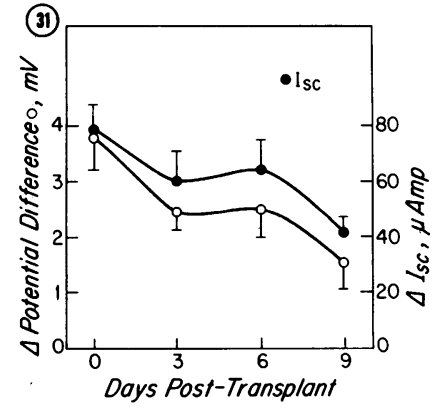

Figure 31. Effect of transplantation on stimulated $\mathrm{Na}^{+}$-coupled glucose transport of graft jejuna as assessed by measurement of electrical responses to $20 \mathrm{mM}$ mucosal glucose. Impaired absorptive responses could be detected by measurements of $I_{\mathrm{sc}}$ at $9 \mathrm{~d}$ or of PD by $3 \mathrm{~d}$. 


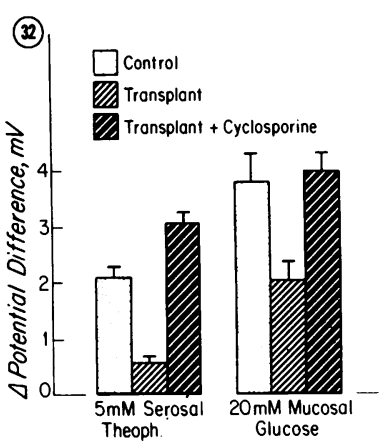

Per unit of actual mucosal surface area, resistance to passive transepithelial ion flow also significantly decreased in allografts both at 6 and $9 \mathrm{~d}$, but not at $3 \mathrm{~d}$, in comparison with host jejuna. Thus, because only minimal epithelial structural injury was present at $3 \mathrm{~d}$, the evolution of the defect in barrier function of allografts correlates well with the temporal evolution of structural epithelial injury.

We also measured parameters of baseline and stimulated active ion transport at 3,6 , and $9 \mathrm{~d}$ in allograft and host jejuna. Comparisons with host jejuna were again used to ensure that alterations in allograft mucosal function were not simply due to nonspecific systemic effects of the illness that accompanies transplant rejection. As with resistance, spontaneous potential difference declined comparably in both host and graft jejuna at $3 \mathrm{~d}$. However, although the spontaneous PD of host jejuna returned to control levels by $6 \mathrm{~d}$, that of graft jejuna progressively fell at 6 and $9 \mathrm{~d}$ finally to reach a level significantly less than either control or 9-d host values. Inasmuch as we previously have shown that 9-d isografts do not show diminished PDs compared with host or to control jejuna (1), the decrease in PDs seen at $9 \mathrm{~d}$ in allografts may be related to rejection and is not simply secondary to the grafting procedure. Further support of this interpretation comes from the finding that cyclosporine therapy can prevent this fall in spontaneous PD of the allograft as measured $9 \mathrm{~d}$ posttransplantation (1). The responses of host jejunal epithelium to agents that stimulate active absorption (mucosal glucose) or active secretion (serosal theophylline) as measured by increments in PD and $I_{\text {sc }}$ were not significantly attenuated at any assessed postoperative time in host jejunum. However, the PD and $I_{s c}$ responses to serosal theophylline (secretion) progressively diminished in allografts as compared with host jejunal epithelium, with major declines occurring between 3-6 and 6-9 d. Inasmuch as electrogenic $\mathrm{Cl}^{-}$secretion, the response that we are assessing with theophylline (6) appears in part, although perhaps not exclusively (2), to originate from the crypt $(2,3$, 6 ), this progressive defect in stimulated secretory response fits well with the progressive structural damage to crypt epithelial cells that we observe between 3 and $9 \mathrm{~d}$. The PD and $I_{\mathrm{sc}}$ responses of allograft epithelium to mucosal glucose (absorption) were substantially diminished as compared with host jejunal epithelium at $9 \mathrm{~d}$ but not at day 3 or day 6 . Because this absorptive response appears to be restricted to villus epithelium (7) and we find no substantial degree of progressive villus epithelial cell structural damage before day 9, these functional findings also fit well with our structural observations. Cyclosporine therapy was able to prevent the functional abnormalities in stimulated transport from developing in allografts. This finding also parallels the remarkable prevention of epithelial (and endothelial) structural injury provided by cyclosporine therapy.

The above data promote our understanding of the functional and structural evolution of jejunal allograft rejection in this model, and they also suggest a mechanism by which small intestinal epithelial function might be monitored during transplant rejection in the future, because in vivo measurements of transepithelial PDs can be performed in humans with alimentary tract disease (17). If future experimental or clinical small intestinal transplants were constructed to expose at least one stoma after the initial transplant surgery, it should be possible to monitor PD and thus assess the efficacy of immunosuppressive therapy by its ameliorating effect on this parameter of intestinal function in vivo. Moreover, by infusing lumenal solutions containing glucose, one might similarly be able to measure a parameter of stimulated active transport.

Mucosal biopsies might also be retrieved through a stoma to assess structural features of rejection. Inasmuch as experimental animals or, potentially in the future, patients might have other systemic disorders that would affect intestinal mucosal structure (i.e., hypotension, bacterial overgrowth of transplanted loops, etc.) it would be important to assess an easily identified and specific marker of rejection-related structural injury in evaluation of such biopsy material. The microvascular endothelial lesion we describe might represent such a marker because, to our knowledge, similar lesions are not seen with common forms of mucosal injury. Furthermore, because such microvascular lesions are most obvious at the interface between the mucosa and submucosa, and this region of the intestinal wall is routinely included with mucosal suction biopsies, evaluation of this structural parameter of intestinal rejection should not be problematic.

Although, as we previously reported (1), cyclosporine therapy can extend the life of a rat harboring an intestinal allograft far beyond $9 \mathrm{~d}$, we do not yet know whether intestinal structure and function is as ideally retained at these longer time periods as it is at $9 \mathrm{~d}$. Adequate evaluation of long-term grafts may be difficult in the present model, inasmuch as the allograft is not in continuity with the host bowel. Long-term exclusion from continuity could result in a variety of secondary alterations to intestinal epithelial cells. For example, the absence of pancreatic proteases might enhance mucosal function by prolonging the half-life of integral membrane proteins of absorptive cell microvillus membranes (18). On the other hand, such an excluded loop provides an ideal setting for the development of bacterial overgrowth (19), which can compromise mucosal structure $(20,21)$ and function $(21,22)$. A variety of other potential factors (lack of microvillus membrane enzyme substrate induction [23], lack of growth promoting luminal bulk [23], etc.) would also complicate detailed analysis of long-term excluded allografts. Fortunately, our previous studies of isografts (1), and current studies of cyclosporine-treated allografts suggest that such potentially confounding factors do not substantially alter the structural and functional parameters we have studied during the immediate 9-d postoperative period.

\section{Acknowledgments}

The authors would like to acknowledge the skilled technical assistance of Susan Carlson. We are also grateful to Mary LoGiudice for preparing this manuscript.

This work was supported by grants AM-33040 and AM-27972 from the National Institutes of Health. 


\section{References}

1. Kirkman, R. L., P. A. Lear, J. L. Madara, and N. L. Tilney. 1984. Small intestine transplantation in the rat-immunology and function. Surgery. 96:280-286.

2. Donowitz, M., and J. L. Madara. 1982. Effect of extracellular calcium depletion on epithelial structure and function in rabbit ileum: a model for selective crypt or villus epithelial cell damage and suggestion of secretion by villus epithelial cells. Gastroenterology. 83:1231-1243.

3. Welsh, M. J., P. L. Smith, M. Fromm, and R. A. Frizzell. 1982. Crypts are the site of intestinal fluid and electrolyte secretion. Science (Wash. DC). 218:1219-1221.

4. Madara, J. L. 1983. Increases in guinea pig intestinal transepithelial resistance induced by osmotic loads are accompanied by rapid alterations in absorptive cell tight junction structure. J. Cell Biol. 97:125-136.

5. Madara, J. L., and J. S. Trier. 1982. Structure and permeability of goblet cell tight junctions in rat small intestine. J. Membr. Biol. 66:145-157.

6. Field, M. 1981. Secretion of electrolytes and water by mammalian small intestine. In Physiology of the Gastrointestinal Tract. L. R. Johnson editor. Raven Press, New York. 963-982.

7. Schultz, S. G. 1978. Ion-coupled transport across biological membranes. In Physiology of Membrane Disorders. T. E. Andreoli, J. R. Hoffman, and D. D. Fanestil, editors. Plenum Press, New York. 273-286.

8. Trier, J. S., and J. L. Madara. 1981. Morphology of the mucosa of the small intestine. In Physiology of the Gastrointestinal Tract. L. R. Johnson, editor. Raven Press, New York. 925-962.

9. Forbes, R. D. C., R. D. Guttmann, M. Gomersall, and J. Hibberd. 1983. A controlled serial ultrastructural tracer study of firstset cardiac allograft rejection in the rat. Evidence that the microvascular epithelium is the primary target of graft destruction. Am. J. Pathol. 111:184-196.

10. Dvorak, H. F., M. Mihm, A. M. Dvorak, B. A. Barnes, E. J. Manseau, and S. J. Galli. 1979. Rejection of first-set skin allografts in man: the microvasculature is the critical target of the immune response. J. Exp. Med. 150:322-332.

11. Anderson, N. D., R. G. Wyllie, and I. J. Shaker. 1977. Pathogenesis of vascular injury in rejecting rat renal allographs. Johns Hopkins Med. J. 144:135-147.
12. Messier, B., and C. P. Leblond. 1960. Cell proliferation and migration as revealed by radioautography after injection of thymidine$\mathrm{H}^{3}$ into male rats and mice. Am. J. Anat. 106:247-265.

13. MacDonald, W. C., J. S. Trier, and N. B. Everett. 1964. Cell proliferation and migration in the stomach, duodenum, and rectum of man: radioautographic studies. Gastroenterology. 46:405-417.

14. Wagner, R., H. Gabbert, and P. Höhn. 1979. Ischemia and postischemic regeneration of the small intestinal mucosa. A light microscopic and autoradiographic study. Virchows Arch. B Cell Pathol. 31:259-276.

15. Sale, G. E., H. M. Shulman, G. B. McDonald, and E. D. Thomas. 1979. Gastrointestinal graft-versus-host disease in man: a clinicopathologic study of the rectal mucosa. Am. J. Surg. Pathol. 3:291-299.

16. Epstein, R. J., G. B. McDonald, G. E. Sale, H. M. Schulman, and E. D. Thomas. 1980. The diagnostic accuracy of the rectal biopsy in acute graft-versus-host-disease: a prospective study of thirteen patients. Gastroenterology. 78:764-771.

17. Orlando, R. C., D. W. Powell, J. C. Bryson, H. B. Kinard, C. N. Carney, J. D. Jones, and E. M. Bozymski. 1982. Esophageal potential difference measurements in esophageal disease. Gastroenterology. 83:1026-1032.

18. Alpers, D. H., and F. J. Tedesco. 1975. The possible role of pancreatic proteases in the turnover of intestinal brush border proteins. Biochim. Biophys. Acta. 401:28-40.

19. Donaldson, R. M. 1978. The blind loop syndrome. In Gastrointestinal Disease. M. Sleisenger and J. Fordtran, editors. W. B. Saunders, Philadelphia. 1094-1103.

20. Toskes, P., R. Gianella, H. Jervis, and W. R. Rout. and A. Takeuchi. 1975. Small intestinal mucosal injury in the experimental blind loop syndrome: light and electron microscopic and histochemical studies. Gastroenterology. 68:1193-1203.

21. Wehman, H. J., F. Lifshitz, and S. Teichberg. 1978. Effects of enteric microbial overgrowth on small intestinal ultrastructure in the rat. Am. J. Gastroenterol. 70:249-258.

22. Jonas, A., C. Krishnan, and G. Forstner. 1978. Pathogenesis of mucosal injury in the blind loop syndrome: Release of dissacharidases from brush border membranes by extracts of bacteria obtained from intestinal blind loops in rats. Gastroenterology. 75:791-795.

23. Karasov, W. H., and J. M. Diamond. 1983. Adaptive regulation of sugar and amino acid transport by vertebrate intestine. Am. J. Physiol. 245:6443-6462. 